\title{
RESIDUAL-FREE BUBBLES FOR ADVECTION-DIFFUSION PROBLEMS: THE GENERAL ERROR ANALYSIS
}

\author{
F. BREZZI, D. MARINI \\ Dipartimento di Matematica and I.A.N.-C.N.R. \\ Via Abbiategrasso 215, 27100 Pavia (Italy) \\ E. SÜLI \\ University of Oxford, Computing Laboratory, \\ Wolfson Building, Parks Road, Oxford OX1 3QD (UK)
}

\begin{abstract}
We develop the general a priori error analysis of residual-free bubble finite element approximations to non-self-adjoint elliptic problems of the form $(\varepsilon A+C) u=f$ subject to homogeneous Dirichlet boundary condition, where $A$ is a symmetric second-order elliptic operator, $C$ is a skew-symmetric first-order differential operator, and $\varepsilon$ is a positive parameter. Optimal-order error bounds are derived in various norms, using piecewise polynomial finite elements of degree $k \geq 1$.
\end{abstract}

\section{Introduction}

Although the paper deals with a slightly more general case, let us consider here, for simplicity, the following model problem: find $u \in H_{0}^{1}(\Omega)$ such that

$$
-\varepsilon \Delta u+u_{x}=f \quad \text { in } \Omega,
$$

where $\Omega$ is a bounded polygonal domain in the plane, $f$ is given in $L_{2}(\Omega)$, and $\varepsilon>0$ is very small compared with the diameter of $\Omega$, so that (1.1) is advection-dominated. Let $\left\{\mathcal{T}_{h}\right\}_{h}$ be a sequence of partitions of $\Omega$ into triangles $T$, let $k \geq 1$ be an integer, and consider the finite element space

$$
W_{h} \equiv W_{h}^{k}\left(\mathcal{T}_{h}, \Omega\right)=\left\{v \in H_{0}^{1}(\Omega):\left.\left.v\right|_{T} \in P_{k}\right|_{T} \text { for each } T \text { in } \mathcal{T}_{h}\right\} .
$$

Here $h$ is a positive discretisation parameter which measures the granularity of the partition $\mathcal{T}_{h}$, and $\left.P_{k}\right|_{T}$ denotes the space of polynomials of degree $\leq k$ on $T$. The usual Galerkin finite element approximation to (1.1) is then

$$
\left\{\begin{array}{l}
\text { find } u_{h}^{G} \in W_{h} \text { such that } \\
\int_{\Omega}\left(\varepsilon \nabla u_{h}^{G} \cdot \nabla v+u_{x}^{G} v\right) \mathrm{d} x=\int_{\Omega} f v \mathrm{~d} x \quad \forall v \in W_{h} .
\end{array}\right.
$$


It is well known that, whenever $\varepsilon / h<<1$, this method is unstable, which manifests itself in large maximum-principle-violating oscillations in the numerical solution. Among several possible remedies for this undesirable feature of the usual Galerkin approximation, the SUPG method ([7], [15]) has attracted considerable attention over the last decade, primarily because of its attractive combination of structural simplicity, generality and the quality of the resulting numerical solution. For problem (1.1) the SUPG method reads

$$
\left\{\begin{array}{l}
\text { find } u_{h}^{S} \in W_{h} \text { such that } \\
\int_{\Omega}\left(\varepsilon \nabla u_{h}^{S} \cdot \nabla v+u_{h, x}^{S} v\right) \mathrm{d} x-S\left(u_{h}^{S}, v\right)=\int_{\Omega} f v \mathrm{~d} x \quad \forall v \in W_{h}, \\
\text { where } S\left(u_{h}^{S}, v\right)=\sum_{T} \tau_{T} \int_{T}\left(-\varepsilon \Delta u_{h}^{S}+u_{h, x}^{S}-f\right)\left(-\varepsilon \Delta v-v_{x}\right) \mathrm{d} x,
\end{array}\right.
$$

and $\tau_{T}$ is a parameter which needs to be chosen suitably. Reasonable rules of thumb for the choice of $\tau_{T}$ can be found, for instance, in [9] and the references therein; the corresponding error analysis (for model problems like (1.1)) is given in [14].

In recent years, the SUPG method has been frequently viewed in a more general context (see, e.g., [1], [2] and the references therein), and appropriate choices for the value of $\tau_{T}$ (or, more generally, for a suitable form of the stabilizing term to be added to (1.3)) found a different, and phylosophically more appealing, justification.

For the particular case of piecewise linear elements, for instance, it was shown that SUPG can be also derived by the so-called residual-free bubble approach (RFB from now on; see [6], [10]) as well as by the local Green's function approach ([12]). The connections between these two approaches were clarified in [2]: both strategies lead precisely to (1.4), with a very specific value for $\tau_{T}$ which can be, therefore, considered as optimal, at least from the theoretical point of view.

Since for $k=1$ the SUPG method and the RFB approach (or its equivalent local Green's function counterpart) yield the same scheme, the results of [14] can be used for the error analysis. However, as it was shown in [4], an analysis based on the residual-free bubble framework can arrive at the same results by means of a completely different procedure, casting a new light on the basic underlying features of the new methodology.

In contrast with the case of $k=1$, for $k>1$ the RFB approach produces a stabilizing term similar but not identical to that of (1.4). As a matter of fact, for $k>1$, (1.4) may be obtained by suitable virtual bubbles (see [1]) - an approach which does not follow from the RFB methodology.

The objective of this paper is then to perform the error analysis of the residualfree bubble method for the case of $k>1$. This can be seen, in a sense, as an extension of [4], although the techniques of error analysis presented here are quite different and, to the best of our knowledge, completely new in this context. They are based on sharp interpolation results in certain Besov spaces of differential order $1 / 2$ which do not coincide with the usual Hilbertian Sobolev spaces $H^{1 / 2}$ or $H_{00}^{1 / 2}$.

The outline of the paper is as follows. In Section 2 we present our model problem, and we recall the basic features of the RFB method applied to it. In Section 3 we 
recall the definitions of the Besov spaces mentioned above and we prove some simple properties of these which will be used in the subsequent analysis. Finally, the error analysis is presented in Section 4 .

Numerical experiments to compare the relative performances of SUPG and RFB for values of $k>1$ would be very interesting but are beyond the scope of this paper and are not discussed here.

\section{Statement of the problem}

Suppose that $\Omega$ is a bounded polyhedral domain in $\mathbf{R}^{n}$ and let $L$ be a second-order linear differential operator of the form

$$
L=\varepsilon A+C,
$$

where $A$ and $C$ are defined, for $w$,say, in $H^{1}(\Omega)$, by

$$
A w \equiv-\sum_{i, j=1}^{n} \frac{\partial}{\partial x_{j}}\left(a_{i j}(x) \frac{\partial w}{\partial x_{i}}\right), \quad C w \equiv \sum_{i=1}^{n} c_{i}(x) \frac{\partial w}{\partial x_{i}} .
$$

We assume that, for almost every $x$ in $\Omega$, the $n \times n$ matrix $\left(a_{i j}(x)\right)$ is symmetric and positive definite, with smallest eigenvalue $\geq \alpha>0$ and largest eigenvalue $\leq 1$, independent of $x$. In a sense, we are normalizing the operator $A$ in the product $\varepsilon A$. To the operator $A$ we assign the bilinear form

$$
a(w, v)=\int_{\Omega} \sum_{i, j=1}^{n} a_{i j}(x) \frac{\partial w}{\partial x_{i}} \frac{\partial v}{\partial x_{j}} \mathrm{~d} x, \quad w, v \in H^{1}(\Omega) .
$$

With the above assumptions $A$ is a symmetric operator from $H_{0}^{1}(\Omega)$ into $H^{-1}(\Omega)$ verifying

$$
a(w, v)=\langle A w, v\rangle=\langle w, A v\rangle \quad \forall w, v \in V,
$$

where, from now on,

$$
V=H_{0}^{1}(\Omega), \quad V^{\prime}=H^{-1}(\Omega),
$$

equipped with respective norms $\|\cdot\|_{H^{1}(\Omega)}$ and $\|\cdot\|_{H^{-1}(\Omega)}$, and $\langle\cdot, \cdot\rangle$ denotes the duality pairing between $V$ and $V^{\prime}$. Moreover, the bilinear form $a(\cdot, \cdot)$ is $V$-elliptic and normalised to 1 , that is,

$$
\begin{gathered}
\alpha|v|_{H^{1}(\Omega)}^{2} \leq a(v, v) \quad \forall v \in V, \\
a(v, w) \leq|v|_{H^{1}(\Omega)}|w|_{H^{1}(\Omega)} \quad \forall v, w \in V,
\end{gathered}
$$

where $|\cdot|_{H^{1}(\Omega)}$ is the seminorm of $V=H_{0}^{1}(\Omega)$.

Similarly, we assume that, for almost every $x$ in $\Omega$, the $n$-component vector $\left(c_{i}(x)\right)$ has Euclidean norm $\leq \gamma$, independent of $x$, and we introduce the bilinear form $c(\cdot, \cdot)$, defined by

$$
c(w, v)=\int_{\Omega} \sum_{i=1}^{n} c_{i}(x) \frac{\partial w}{\partial x_{i}} v \mathrm{~d} x, \quad w \in H^{1}(\Omega), v \in L_{2}(\Omega) .
$$


As a consequence, we have

$$
c(w, v)=(C w, v) \quad \forall w \in H^{1}(\Omega), \forall v \in L_{2}(\Omega),
$$

where $(\cdot, \cdot)$ signifies the inner product in $L_{2}(\Omega)$. Our hypotheses imply that

$$
|c(w, v)| \leq \gamma|w|_{H^{1}(\Omega)}\|v\|_{L_{2}(\Omega)} \quad \forall w \in H^{1}(\Omega), \forall v \in L_{2}(\Omega),
$$

where $\|\cdot\|_{L_{2}(\Omega)}$ is the norm of $L_{2}(\Omega)$. We make the additional assumption that the bilinear form $c(\cdot, \cdot)$ is skew-symmetric on $V$; namely,

$$
c(w, v)=-c(v, w)=(C w, v)=-(w, C v) \quad \forall w, v \in V .
$$

This can be ensured by requiring that the vector field $\mathbf{c}=\left(c_{1}, \ldots, c_{n}\right)$ is divergencefree on $\Omega$ in the sense of distributions.

For $f$ given in $L_{2}(\Omega)$, say, we consider the boundary value problem

$$
\left\{\begin{aligned}
L u=f & \text { in } \Omega, \\
u=0 & \text { on } \partial \Omega .
\end{aligned}\right.
$$

Let $\mathcal{L}(\cdot, \cdot)$ be the bilinear form on $V \times V$ associated with the operator $L$, namely,

$$
\mathcal{L}(w, v)=\varepsilon a(w, v)+c(w, v) \quad \forall w, v \in V .
$$

We consider the variational form of (2.10):

$$
\left\{\begin{array}{l}
\text { find } u \in V \text { such that } \\
\mathcal{L}(u, v)=(f, v) \quad \forall v \in V .
\end{array}\right.
$$

Applying (2.11), (2.4) and (2.9), it is easy to check that

$$
\alpha \varepsilon|v|_{H^{1}(\Omega)}^{2} \leq \mathcal{L}(v, v) \quad \forall v \in V .
$$

By virtue of (2.13) and the Lax-Milgram lemma, (2.12) has a unique solution in $V$.

Next we formulate the RFB-approximation of (2.12). Suppose that we are given a shape-regular family of partitions $\left\{\mathcal{T}_{h}\right\}_{h}$ of $\Omega$ into open $n$-simplices $T$ (referred to as elements), and an integer $k \geq 1$. We recall that $\left\{\mathcal{T}_{h}\right\}_{h}$ is said to be a shaperegular family if there exists a fixed positive constant $\mu$ such that, for each $\mathcal{T}_{h}$ and each $T \in \mathcal{T}_{h}$,

$$
\frac{h_{T}}{\rho_{T}} \leq \mu,
$$

where $h_{T}$ denotes the diameter of the $n$-simplex $T$ (i.e. its longest edge), and $\rho_{T}$ is the diameter of the largest ball inscribed in $T$. We set

$$
\begin{gathered}
V_{h} \equiv V_{h}^{k}\left(\mathcal{T}_{h}, \Omega\right)=\left\{v \in V:\left.\left.v\right|_{e} \in P_{k}\right|_{e} \text { for each }(n-1)\right. \text {-dimensional } \\
\text { face } \left.e \text { of any element } T \text { in the partition } \mathcal{T}_{h}\right\}
\end{gathered}
$$

Here $\left.P_{k}\right|_{e}$ denotes the set of all polynomials in $(n-1)$ variables of degree $\leq k$ on the face (or edge for $n=2$ ) $e$. The discrete counterpart of (2.12) is then 


$$
\left\{\begin{array}{l}
\text { find } u_{h} \in V_{h} \text { such that } \\
\mathcal{L}\left(u_{h}, v_{h}\right)=\left(f, v_{h}\right) \quad \forall v_{h} \in V_{h} .
\end{array}\right.
$$

Notice that $V_{h}$ is not the usual finite element space of continuous piecewise polynomial functions (that would be the space $W_{h}$ defined in (1.2)), but can be thought of as being obtained by supplementing $W_{h}$ by the space of all functions in $H_{0}^{1}(T)$, for all $T$ in $\mathcal{T}_{h}$. More precisely,

$$
V_{h}=W_{h}+B_{h},
$$

where

$$
B_{h}=\bigoplus_{T \in \mathcal{T}_{h}} H_{0}^{1}(T)
$$

In particular, $V_{h}$ is not finite-dimensional. In the following discussion we shall show that problem (2.16) is equivalent to a finite-dimensional one. However, working on formulation (2.16) makes the analysis simpler. For instance we can immediately point out that, for every $T \in \mathcal{T}_{h}$, and for every $\varphi \in C_{0}^{\infty}(T)$, it is possible to construct $v_{h} \in V_{h}$ by selecting $v_{h}=\varphi$ in $T$, and $v_{h}$ identically zero outside $T$. Consequently, from (2.16), we conclude easily that

$$
L u_{h}=f \quad \text { in each } T \text { in } \mathcal{T}_{h},
$$

which is the property that justifies the name residual-free.

In the remaining part of this Section we shall analyse (2.16) from the point of view of the possible computational techniques. In doing so, we shall also clarify its relationships with the SUPG-method.

We start the analysis with the following considerations, typical of the RFBapproach (see, e.g., [2]). The solution $u_{h}$ of (2.19) is a polynomial of degree $k$ on each $e$ of $\partial T$ (see (2.15)). Let $p_{k}$ be a polynomial of degree $\leq k$ in $T$ having the same boundary value (on $\partial T$ ) as $u_{h}$. Such a polynomial is not unique for $k>n$, but this is not essential. Then,

$$
u_{h}=p_{k}+u_{b}
$$

where $u_{b} \in H_{0}^{1}(T)$ and, using (2.19), solves

$$
L_{T} u_{b}=-L p_{k}+f \quad \text { in each } T \text { in } \mathcal{T}_{h} .
$$

where $L_{T}: H_{0}^{1}(T) \rightarrow H^{-1}(T)$ denotes the restriction of the operator $L$ to $T$; namely, $L_{T} w=L w$ for all $w \in H_{0}^{1}(T), T \in \mathcal{T}_{h}$. Notice that $L_{T}$ is injective, so that (2.21) can be written as

$$
u_{b}=L_{T}^{-1}\left(-L p_{k}+f\right) \quad \text { in each } T \text { in } \mathcal{T}_{h} .
$$

Assume now that $f$ is a piecewise polynomial of degree $\leq(k-1)$, and $A$ and $C$ have piecewise constant coefficients. Then, in each $T$, the right-hand side of (2.21) is a 
polynomial of degree $\leq(k-1)$, and consequently $u_{b}$ must belong to the subspace $B(T)$ of $H_{0}^{1}(T)$ made up of all possible solutions of $L_{T} u_{b}=g$ when $g$ ranges through $P_{k-1}$, that is,

$$
B(T)=\left\{v_{b} \in H_{0}^{1}(T): \quad L v_{b} \in P_{k-1}(T)\right\} .
$$

Clearly $B(T)$ is a finite-dimensional space, and its dimension is bounded by

$$
\left(\begin{array}{c}
k-1+n \\
n
\end{array}\right) \text {. }
$$

Finally, from (2.20) we deduce that, for all $T,\left.u_{h}\right|_{T}$ belongs to $P_{k}+B(T)$, so that $u_{h}$ belongs to the space $\widetilde{V}_{h}$ defined as

$$
\widetilde{V}_{h}=W_{h} \oplus \widetilde{B}_{h},
$$

where

$$
\widetilde{B}_{h}=\bigoplus_{T \in \mathcal{T}_{h}} B(T) .
$$

and $B(T)$ is still given by (2.23). In particular, $u_{h}$ will coincide with the unique solution $\widetilde{u}_{h}$ of the problem

$$
\left\{\begin{array}{l}
\text { find } \widetilde{u}_{h} \in \widetilde{V}_{h} \text { such that } \\
\mathcal{L}\left(\widetilde{u}_{h}, v\right)=(f, v) \quad \forall v \in \widetilde{V}_{h} .
\end{array}\right.
$$

As problem (2.24) is clearly finite-dimensional, we can see that (2.16) is equivalent to a finite-dimensional problem. For low $k$, a set of basis functions for $B(T)$ can be computed approximately, at a reasonable cost, at least for $\varepsilon<<h_{T}$, where $h_{T}=\operatorname{diam}(T)$ (see [6], [10], [3] [5], [2] for various developments of this idea). The elements of $B(T)$ are referred to as residual-free bubbles. A possible variant of (2.24), although not equivalent to (2.16), is to choose $B(T)$, on each element $T$, as a finite dimensional subspace of $H_{0}^{1}(T)$ such that $B(T) \cap P_{k}=\{0\}$, the selection of $B(T)$ being guided by the desire to resolve features of the analytical solution which could not otherwise be captured by seeking a numerical solution from the classical finite element space $W_{h}$. In fact, such an enhancement of $W_{h}$ achieves more than merely improving the 'resolution of fine scales', as we shall now show by investigating the relationships of (2.16) with the SUPG approach.

Going back to the splitting (2.17), we can decompose $u_{h}$ as

$$
u_{h}=u_{k}+u_{b}, \quad u_{k} \in W_{h}, \quad u_{b} \in B_{h} .
$$

We observe at this point that $V_{h}$ is not a direct sum of $W_{h}$ and $B_{h}$, and therefore the decomposition (2.25) of $u_{h}$ is non-unique. In practice, this conceptual difficulty does not arise, since, as already pointed out, instead of operating on the whole of $V_{h}$ one would be computing on its finite-dimensional subspace $\widetilde{V}_{h}$. On inserting the decomposition (2.25) into (2.16), and choosing $v_{h}=v_{k} \in W_{h}$ and $v_{h}=v_{b} \in B_{h}$, we obtain, respectively,

$$
\begin{aligned}
\mathcal{L}\left(u_{k}, v_{k}\right)+\mathcal{L}\left(u_{b}, v_{k}\right) & =\left(f, v_{k}\right) \quad \forall v_{k} \in W_{h}, \\
\mathcal{L}\left(u_{k}, v_{b}\right)+\mathcal{L}\left(u_{b}, v_{b}\right) & =\left(f, v_{b}\right) \quad \forall v_{b} \in B_{h} .
\end{aligned}
$$


As $w=\left.v_{b}\right|_{T} \in H_{0}^{1}(T)$ for each $T$ in $\mathcal{T}_{h}$, after integration by parts (2.27) can be rewritten in the form

$$
\left(L u_{b}, w\right)_{T}=\left(f-L u_{k}, w\right)_{T} \quad \forall w \in H_{0}^{1}(T), \quad T \in \mathcal{T}_{h} .
$$

As we already saw in (2.22), equation (2.28) implies that

$$
u_{b}=L_{T}^{-1}\left(f-L u_{k}\right) .
$$

Substituting this into (2.26) and recalling from (2.1) that $L=C+\varepsilon A$, we find that

$$
\begin{aligned}
& \mathcal{L}\left(u_{k}, v_{k}\right)+\sum_{T \in \mathcal{T}_{h}}\left(L_{T}^{-1}(C+\varepsilon A) u_{k},(C-\varepsilon A) v_{k}\right)_{T} \\
& =\left(f, v_{k}\right)+\sum_{T}\left(L_{T}^{-1} f,(C-\varepsilon A) v_{k}\right)_{T} \quad \forall v_{k} \in W_{h} .
\end{aligned}
$$

Now, (2.29) can be viewed as a stabilised finite element approximation of the convection-diffusion problem (2.12) over the standard finite element space $W_{h}$. A particularly simple case is when $k=1$; then $A u_{k}=0$ and $A v_{k}=0$ for each $v_{k} \in W_{k}$. Moreover, if $f$ and the coefficients of $C$ are piecewise constant, the space $B(T)$, defined in (2.23), has dimension one. Therefore (2.29) reduces to the SUPG method; see [4] for further details.

\section{The functional analytic setting}

In this section we introduce the function spaces and norms which will be used below in the error analysis. Let $T$ be an element in $\mathcal{T}_{h}$ and let $h_{T}$ denote the diameter of $T$. We recall the definitions of some Besov spaces on $T$; of course, these definitions still hold more generally when the element $T$ is replaced by a bounded open set with, say, Lipschitz-continuous boundary. In tandem with the usual natural norm $\|\cdot\|_{H^{1}(T)}$ we shall use in $H^{1}(T)$ the equivalent norm $|[\cdot]|_{H^{1}(T)}$ defined by

$$
|[v]|_{H^{1}(T)}^{2}=h_{T}^{-2}\|v\|_{L_{2}(T)}^{2}+|v|_{H^{1}(T)}^{2} .
$$

For $v \in \Sigma(T):=L_{2}(T)+H^{1}(T)\left(\equiv L_{2}(T)\right)$ and $t>0$, we set

$$
\begin{gathered}
K(t, v)=\inf _{\substack{v=v_{0}+v_{1} \\
v_{0} \in L_{2}(T), v_{1} \in H^{1}(T)}}\left\{\left\|v_{0}\right\|_{L_{2}(T)}+t\left|\left[v_{1}\right]\right|_{H^{1}(T)}\right\} .
\end{gathered}
$$

Similarly, for any $v \in \Sigma_{0}(T):=L_{2}(T)+H_{0}^{1}(T)\left(\equiv L_{2}(T)\right)$ and $t>0$, we define

$$
\begin{gathered}
K_{0}(t, v)=\inf _{\substack{v=v_{0}+v_{1} \\
v_{0}}}\left\{\left\|L_{2}(T), v_{1} \in v_{0}^{1}\right\|_{L_{2}(T)}+t\left|v_{1}\right|_{H^{1}(T)}\right\} .
\end{gathered}
$$

Following the notation of [17] we introduce now the function spaces

$$
\begin{aligned}
X(\infty, T) & =\left\{v \in \Sigma_{0}(T): t \mapsto t^{-1 / 2} K_{0}(t, v) \in L_{\infty}(0, \infty)\right\}, \\
Y(1, T) & =\left\{v \in \Sigma(T): t \mapsto t^{-3 / 2} K(t, v) \in L_{1}(0, \infty)\right\},
\end{aligned}
$$


with respective norms $\|\cdot\|_{X(\infty, T)}$ and $\|\cdot\|_{Y(1, T)}$, defined by

$$
\begin{aligned}
\|v\|_{X(\infty, T)} & =\left\|t^{-1 / 2} K_{0}(\cdot, v)\right\|_{L_{\infty}(0, \infty)} \\
\|v\|_{Y(1, T)} & =\left\|t^{-3 / 2} K(\cdot, v)\right\|_{L_{1}(0, \infty)} .
\end{aligned}
$$

Alternatively, exploiting the interpolation functor $(\cdot, \cdot)_{\theta, p}, 0<\theta<1,1 \leq p \leq \infty$, of the $K$-method of function space interpolation, $X(\infty, T)$ and $Y(1, T)$ can be identified with certain Besov spaces:

$$
\begin{aligned}
X(\infty, T) & =\left(L_{2}(T), H_{0}^{1}(T)\right)_{1 / 2, \infty}=\stackrel{\circ}{B}, 1 / \infty_{2, \infty}(T), \\
Y(1, T) & =\left(L_{2}(T), H^{1}(T)\right)_{1 / 2,1}=B_{2,1}^{1 / 2}(T) .
\end{aligned}
$$

We have the following result.

Proposition 1 Let $T$ be an element in $\mathcal{T}_{h}$. Then, for every $v$ in $X(\infty, T)$ and every $t$ with $0<t<\infty$, there exist $v_{0} \in L_{2}(T)$ and $v_{1} \in H_{0}^{1}(T)$ with $v=v_{0}+v_{1}$ such that

$$
t^{-1 / 2}\left\|v_{0}\right\|_{L_{2}(T)}+t^{1 / 2}\left|v_{1}\right|_{H^{1}(T)} \leq 2\|v\|_{X(\infty, T)} .
$$

Proof: The result follows immediately from (3.6) and the definition of $K_{0}(t, v)$ given in (3.3).

We now consider the master element

$$
\widehat{T}=\left\{\widehat{x}=\left(\widehat{x}_{1}, \ldots, \widehat{x}_{n}\right) \in \mathbf{R}^{n}: \widehat{x}_{i}>0, i=1, \ldots, n, \widehat{x}_{1}+\ldots+\widehat{x}_{n}<1\right\}
$$

and the affine transformation $\widehat{x} \in \widehat{T} \mapsto x=M \widehat{x}+N$ where $M$ is an $n \times n$ matrix and $N$ is an $n \times 1$ column vector. Given a function $v$ defined on $T$, we introduce the function $\widehat{v}$ on $\widehat{T}$ by $\widehat{v}(\widehat{x})=v(x)$. Since the partition $\mathcal{T}_{h}$ is shape regular (c.f. (2.14)), there exist positive constants $\beta_{1}=\beta_{1}(\mu)$ and $\beta_{2}=\beta_{2}(\mu)$ such that [8]

$$
\begin{gathered}
\beta_{1} h_{T}^{n / 2}\|\widehat{v}\|_{L_{2}(\widehat{T})} \leq\|v\|_{L_{2}(T)} \leq \beta_{2} h_{T}^{n / 2}\|\widehat{v}\|_{L_{2}(\widehat{T})}, \quad v \in L_{2}(T), \\
\beta_{1} h_{T}^{n / 2-1}|\widehat{v}|_{H^{1}(\widehat{T})} \leq|v|_{H^{1}(T)} \leq \beta_{2} h_{T}^{n / 2-1}|\widehat{v}|_{H^{1}(\widehat{T})}, \quad v \in H^{1}(T) .
\end{gathered}
$$

From (3.10)-(3.11) we can deduce the analogous inequalities for other norms. In particular, we immediately have for the norm (3.1)

$$
\beta_{1} h_{T}^{n / 2-1}|[\widehat{v}]|_{H^{1}(\widehat{T})} \leq|[v]|_{H^{1}(T)} \leq \beta_{2} h_{T}^{n / 2-1}|[\widehat{v}]|_{H^{1}(\widehat{T})}, \quad v \in H^{1}(T) .
$$

Similarly, with some additional computation we can easily deduce:

$$
\begin{gathered}
\beta_{1} h_{T}^{(n-1) / 2}\|\widehat{v}\|_{X(\infty, \widehat{T})} \leq\|v\|_{X(\infty, T)} \leq \beta_{2} h_{T}^{(n-1) / 2}\|\widehat{v}\|_{X(\infty, \widehat{T})}, \quad v \in X(\infty, T), \\
\beta_{1} h_{T}^{(n-1) / 2}\|\widehat{v}\|_{Y(1, \widehat{T})} \leq\|v\|_{Y(1, T)} \leq \beta_{2} h_{T}^{(n-1) / 2}\|\widehat{v}\|_{Y(1, \widehat{T})}, \quad v \in Y(1, T) .
\end{gathered}
$$

For instance, a typical crucial step is

$$
\begin{aligned}
& t^{-1 / 2} h_{T}^{-n / 2}\left\|v_{0}\right\|_{L_{2}(T)}+t^{1 / 2} h_{T}^{-n / 2+1}\left|v_{1}\right|_{H^{1}(T)} \\
& =h_{T}^{(1-n) / 2}\left\{t^{-1 / 2} h_{T}^{-1 / 2}\left\|v_{0}\right\|_{L_{2}(T)}+t^{1 / 2} h_{T}^{1 / 2}\left|v_{1}\right|_{H^{1}(T)}\right\} \\
& =h_{T}^{(1-n) / 2}\left\{\tau^{-1 / 2}\left\|v_{0}\right\|_{L_{2}(T)}+\tau^{1 / 2}\left|v_{1}\right|_{H^{1}(T)}\right\},
\end{aligned}
$$


with $\tau=t h_{T}$. Finally, denoting, as usual, by $(X(\infty, T))^{\prime}$ the dual space of $X(\infty, T)$ and using the definition of dual norm we have

$$
\beta_{1} h_{T}^{(n+1) / 2}\|\widehat{v}\|_{(X(\infty, \widehat{T}))^{\prime}} \leq\|v\|_{(X(\infty, T))^{\prime}} \leq \beta_{2} h_{T}^{(n+1) / 2}\|\widehat{v}\|_{(X(\infty, \widehat{T}))^{\prime}} .
$$

Using the above inequalities, one can show that the various constants which arise in our analysis below are independent of the diameter $h_{T}$ of the element $T$ considered. In particular, we have the following Gagliardo-Nirenberg type inequality.

Proposition 2 Suppose that $T$ is an element in $\mathcal{T}_{h}$. Then there exists a positive constant $\beta_{0}=\beta_{0}(\mu)$, independent of $T$, such that

$$
\|v\|_{Y(1, T)} \leq \beta_{0}\|v\|_{L_{2}(T)}^{1 / 2}|[v]|_{H^{1}(T)}^{1 / 2}
$$

for each $v$ in $H^{1}(T)$ and each $T \in \mathcal{T}_{h}$.

Proof: From (3.8), with $T$ replaced by the master element $\widehat{T}$, we have that $Y(1, \widehat{T})=\left(L_{2}(\widehat{T}), H_{0}^{1}(\widehat{T})\right)_{1 / 2,1}$; thus, by Theorem 1.3.3(g) in Triebel [18],

$$
\|\widehat{v}\|_{Y(1, \widehat{T})} \leq c_{0}\|\widehat{v}\|_{L_{2}(\widehat{T})}^{1 / 2}\|\widehat{v}\|_{H^{1}(\widehat{T})}^{1 / 2} \quad \forall \widehat{v} \in H^{1}(\widehat{T}),
$$

where $c_{0}$ is a fixed positive constant. On returning from $\widehat{x} \in \widehat{T}$ to our original variable $x \in T$, with $v(x)=\widehat{v}(\widehat{x})$, using (3.14), (3.18), (3.10)-(3.11), and (3.1), the estimate (3.17) follows. $\diamond$

Now we apply a deep result from the theory of function space interpolation due to Tartar [17].

Proposition 3 Suppose that $T$ is an element in $\mathcal{T}_{h}$. Then there exists a positive constant $\beta_{0}=\beta(\mu)$ (possibly different than in Proposition 2), independent of $T$, such that

$$
\|v\|_{X(\infty, T)} \leq \beta_{0}\|v\|_{Y(1, T)} .
$$

for each $v$ in $Y(1, T)$ and each $T$ in $\mathcal{T}_{h}$.

Proof: By Theorem 5 in the work of Tartar [17], $Y(1, \widehat{T}) \subset X(\infty, \widehat{T})$ with continuous embedding; thus, there exists a fixed positive constant $c_{0}$ (possibly different than in (3.18)), such that

$$
\|\widehat{v}\|_{X(\infty, \widehat{T})} \leq c_{0}\|\widehat{v}\|_{Y(1, \widehat{T})} \quad \forall \widehat{v} \in Y(1, \widehat{T}) .
$$

Applying (3.14) and (3.13) we deduce (3.19), with $\beta_{0}=c_{0} \beta_{2} / \beta_{1}$. $\diamond$

We emphasise here that, as (for instance) $H^{1}(T) \subset Y(1, T)$, the result of Proposition 3 implies

$$
H^{1}(T) \subset Y(1, T) \subset X(\infty, T) .
$$

We end this section with an inverse-type inequality which will be useful in the next section. 
Proposition 4 Let $T$ be an element in $\mathcal{T}_{h}$, and let $k$ be a non-negative integer. There exist two positive constants $\beta_{1}=\beta_{1}(\mu, k)$, and $\beta_{2}=\beta_{2}(\mu, k)$, independent of $T$, such that, for every $w$ in $P_{k}(T)$,

$$
\beta_{1} h_{T}^{1 / 2}\|w\|_{L_{2}(T)} \leq\|w\|_{(X(\infty, T))^{\prime}} \leq \beta_{2} h_{T}^{1 / 2}\|w\|_{L_{2}(T)} .
$$

Proof: The proof follows easily from (3.10) and (3.16) by a standard scaling argument (see, e.g., [8]) and exploiting the equivalence of norms on $P_{k}(\widehat{T})$.

\section{Error analysis}

Now we embark on the a priori error analysis of the method (2.16). As a first step we need the basic Lemma stated below. We shall use the notation

$$
\||w|\|_{T}^{2}=\gamma_{T}\|w\|_{L_{2}(T)}|[w]|_{H^{1}(T)}, \quad w \in H^{1}(T),
$$

where

$$
\gamma_{T}=\left(\sum_{j=1}^{n}\left\|c_{j}\right\|_{L_{\infty}(T)}^{2}\right)^{1 / 2}
$$

and we put

$$
\|w\|\left\|^{2}=\sum_{T}\right\| \mid w \|_{T}^{2}, \quad w \in H^{1}(\Omega) .
$$

Lemma 1 There exists a positive constant $\beta=\beta(\mu)$, such that, for every $\psi$ in $H^{1}(\Omega)$, and every $\varphi$ in $H^{1}(\Omega)$ satisfying

$$
\varepsilon A \varphi+C \varphi=0 \quad \text { in } T \quad \text { for every } T \in \mathcal{T}_{h},
$$

we have

$$
|(\psi, C \varphi)| \leq \beta \varepsilon^{1 / 2}|\varphi|_{H^{1}(\Omega)}|||\psi||| .
$$

Proof: Consider $\psi \in H^{1}(\Omega)$ and $\varphi \in H^{1}(\Omega)$, as in the statement of the Lemma. Let us first show that, for every $T \in \mathcal{T}_{h}$, we have

$$
\left|(\psi, C \varphi)_{T}\right| \leq 2\left(\varepsilon \gamma_{T}\right)^{1 / 2}|\varphi|_{H^{1}(T)}\|\psi\|_{X(\infty, T)},
$$

where $(\cdot, \cdot)_{T}$ denotes the inner product of $L_{2}(T)$. Applying Proposition 1 with $t=\left(\varepsilon / \gamma_{T}\right)$ and $v=\left.\psi\right|_{T} \in H^{1}(T) \subset X(\infty, T)$ (c.f. (3.20)) we deduce the existence of $\psi_{0}$ and $\psi_{1}$, with $\psi_{1} \in H_{0}^{1}(T)$ such that

$$
\psi=\psi_{0}+\psi_{1} \quad \text { on } T
$$

and

$$
\left(\frac{\varepsilon}{\gamma_{T}}\right)^{-1 / 2}\left\|\psi_{0}\right\|_{L_{2}(T)}+\left(\frac{\varepsilon}{\gamma_{T}}\right)^{1 / 2}\left\|\psi_{1}\right\|_{H^{1}(T)} \leq 2\|\psi\|_{X(\infty, T)}
$$


Further, since $C \varphi=-\varepsilon A \varphi \in L_{2}(T)$ and $\psi_{1} \in H_{0}^{1}(T)$, we can apply Green's identity to deduce that

$$
\left(\psi_{1}, C \varphi\right)_{T}=-\varepsilon\left(\psi_{1}, A \varphi\right)_{T}=-\varepsilon \sum_{i, j=1}^{n}\left(\frac{\partial \psi_{1}}{\partial x_{j}}, a_{i j} \frac{\partial \varphi}{\partial x_{i}}\right)_{T},
$$

and therefore, using (2.5),

$$
\left|\left(\psi_{1}, C \varphi\right)_{T}\right| \leq \varepsilon\left|\psi_{1}\right|_{H^{1}(T)}|\varphi|_{H^{1}(T)} .
$$

Now, applying (4.3), (2.8), (4.6) and (4.4) we have

$$
\begin{aligned}
& \left|(\psi, C \varphi)_{T}\right|=\left|\left(\psi_{0}, C \varphi\right)_{T}+\left(\psi_{1}, C \varphi\right)_{T}\right| \\
& \quad \leq \gamma_{T}\left\|\psi_{0}\right\|_{L_{2}(T)}|\varphi|_{H^{1}(T)}+\varepsilon\left|\psi_{1}\right|_{H^{1}(T)}|\varphi|_{H^{1}(T)} \\
& \quad \leq\left(\varepsilon \gamma_{T}\right)^{1 / 2}\left\{\left(\frac{\varepsilon}{\gamma_{T}}\right)^{-1 / 2}\left\|\psi_{0}\right\|_{L_{2}(T)}+\left(\frac{\varepsilon}{\gamma_{T}}\right)^{1 / 2}\left\|\psi_{1}\right\|_{H^{1}(T)}\right\}|\varphi|_{H^{1}(T)} \\
& \quad \leq 2\left(\varepsilon \gamma_{T}\right)^{1 / 2}|\varphi|_{H^{1}(T)}\|\psi\|_{X(\infty, T)}
\end{aligned}
$$

that is, (4.2). Using (3.19) and (3.17) in (4.2), we immediately have

$$
\left|(\psi, C \varphi)_{T}\right| \leq \beta \varepsilon^{1 / 2}|\varphi|_{H^{1}(T)}|||\psi| \|_{T} .
$$

Finally, summation over all $T$ and the Cauchy-Schwarz inequality give the result. $\diamond$

We define

$$
u^{I}=\text { quasi interpolant of } u \in H_{0}^{1}(\Omega) \text { from } W_{h} .
$$

For a definition of the quasi-interpolant and the associated error analysis we refer for instance to the recent work of Verfürth [19] and references therein. We then set

$$
\begin{aligned}
& e=u-u_{h}, \\
& \eta=u-u^{I},
\end{aligned}
$$

and note that, from (2.19),

$$
L e=0 \quad \text { in each } T \text { in } \mathcal{T}_{h} .
$$

Moreover, from (2.12), (2.16) we have the usual Galerkin property

$$
\mathcal{L}\left(u-u_{h}, v_{h}\right)=0 \quad \forall v_{h} \in V_{h} .
$$

Thereby, using (4.10) and (4.11),

$$
\mathcal{L}(e, \eta-e)=0 .
$$

From the above Lemma we immediately deduce the following result. 
Theorem 1 Let $u$ and $u_{h}$ be the solutions of (2.12) and (2.16), respectively, and let $e$ and $\eta$ be defined through (4.9)-(4.11). Then there exists a constant $\beta^{*}>0$, independent of $h, \alpha, \gamma$ and $\varepsilon$, such that

$$
\alpha^{2} \varepsilon|e|_{H^{1}(\Omega)}^{2} \leq \beta^{*}\left(\varepsilon|\eta|_{H^{1}(\Omega)}^{2}+\left.|||\eta|\right|^{2}\right) .
$$

Proof: Using (2.13), (4.13) and (2.11), we have that

$$
\alpha \varepsilon|e|_{H^{1}(\Omega)}^{2} \leq \mathcal{L}(e, e)=\mathcal{L}(e, \eta)=\varepsilon a(e, \eta)+c(e, \eta)
$$

From (4.14), using (2.5) and (2.7), it follows that

$$
\alpha \varepsilon|e|_{H^{1}(\Omega)}^{2} \leq \varepsilon|\eta|_{H^{1}(\Omega)}|e|_{H^{1}(\Omega)}+|(\eta, C e)| .
$$

Noting (4.12) and applying (4.1) with $\varphi=e$ and $\psi=\eta$ to the second term on the right-hand side of (4.15), we deduce that

$$
\alpha \varepsilon|e|_{H^{1}(\Omega)}^{2} \leq \varepsilon^{1 / 2}|e|_{H^{1}(\Omega)}\left\{\varepsilon^{1 / 2}|\eta|_{H^{1}(\Omega)}+\beta|\|\eta|\||\}\right.
$$

and the result easily follows.

We note now that, as we have already seen in [4] for the case of $k=1$, the norm

$$
\varepsilon^{1 / 2}|e|_{H^{1}(\Omega)}
$$

is stronger than it might appear at the first sight. Actually, one has the following result.

Theorem 2 Let $u$ and $u_{h}$ be the solutions of (2.12) and (2.16), respectively, and let $e=u-u_{h}$. Then,

$$
\|C e\|_{*}^{2} \leq 4 \varepsilon|e|_{H^{1}(\Omega)}^{2},
$$

where

$$
\|w\|_{*}^{2}=\sum_{T} \frac{1}{\gamma_{T}}\|w\|_{(X(\infty, T))^{\prime}}^{2} .
$$

Proof: From (4.2) one obtains, for every smooth function $\psi$,

$$
\left|(\psi, C e)_{T}\right| \leq 2\left(\varepsilon \gamma_{T}\right)^{1 / 2}|e|_{H^{1}(T)}|| \psi \|_{X(\infty, T)},
$$

which yields

$$
\|C e\|_{(X(\infty, T))^{\prime}} \leq 2\left(\varepsilon \gamma_{T}\right)^{1 / 2}|e|_{H^{1}(T)},
$$

and taking the square

$$
\|C e\|_{(X(\infty, T))^{\prime}}^{2} \leq 4 \varepsilon \gamma_{T}|e|_{H^{1}(T)}^{2} .
$$

Upon summation over all $T$ in $\mathcal{T}_{h}$, this gives the desired inequality. $\diamond$

We note that $\|\cdot\|_{*}$ can be thought of as a sort of broken norm on a space with negative-order $1 / 2$. Its nature is further clarified by our next result. 
Lemma 2 Let $T$ be an element in $\mathcal{T}_{h}$, and let $k$ be a non negative integer. There exist two positive constants $\beta_{1}=\beta_{1}(\mu, k)$, and $\beta_{2}=\beta_{2}(\mu, k)$ independent of $T$, such that,

$$
\beta_{1}\|w\|_{*} \leq\left(\sum_{T} \frac{h_{T}}{\gamma_{T}}\|w\|_{L_{2}(T)}^{2}\right)^{1 / 2} \leq \beta_{2}\|w\|_{*}
$$

for each $w$ in $W_{h}$.

PROOF: The proof follows immediately from Proposition 4 and the definition of the norm $\|\cdot\|_{*}$. $\diamond$

We complete the error analysis by recalling from [19] the following standard local approximation results for the quasi interpolant: there exists a positive constant $\beta=\beta(\mu, k)$ such that for each $\mathcal{T}_{h}$ and each $T$ in $\mathcal{T}_{h}$,

$$
\begin{aligned}
\| u-\left.u^{I}\right|_{L_{2}(T)} & \leq \beta h_{T}^{r+1}|u|_{H^{r+1}(S(T))}, \\
\left|u-u^{I}\right|_{H^{1}(T)} & \leq \beta h_{T}^{r}|u|_{H^{r+1}(S(T))},
\end{aligned}
$$

for $0 \leq r \leq k$, where $h_{T}=\operatorname{diam}(T)$ and $S(T)$ is the union of all elements in $\mathcal{T}_{h}$ whose closure intersects the closure of $T$.

Theorem 3 Let $u$ and $u_{h}$ be the solutions of (2.12) and (2.16), respectively. Assuming that $u \in H^{k+1}(\Omega) \cap H_{0}^{1}(\Omega)$, there exists a positive constant $\beta^{*}=\beta^{*}(\mu, k)$, independent of $h, \alpha, \gamma$ and $\varepsilon$, such that

$$
\begin{aligned}
\varepsilon^{1 / 2} \mid u & -\left.u_{h}\right|_{H^{1}(\Omega)}+\left\|C\left(u-u_{h}\right)\right\|_{*} \\
\leq & \frac{\beta^{*}}{\alpha}\left(\sum_{T \in \mathcal{T}_{h}}\left(\varepsilon h_{T}^{2 r}+\gamma_{T} h_{T}^{2 r+1}\right)|u|_{H^{r+1}(S(T))}^{2}\right)^{1 / 2},
\end{aligned}
$$

where $0 \leq r \leq k$.

Proof: The result follows from Theorem 1, on noting that $\eta=u-u^{I}$ and applying (4.18) and (4.19).

Suppose, in particular, that there is a fixed constant $\mu_{1}>0$ such that, on each element $T$, the local mesh Peclet number

$$
P e_{T}=\frac{\gamma_{T} h_{T}}{\varepsilon} \geq \mu_{1}
$$

We note that this is a reasonable assumption when the problem (2.1) is convectiondominated. Under this hypothesis, it follows from (4.20) that

$$
\varepsilon^{1 / 2}\left|u-u_{h}\right|_{H^{1}(\Omega)} \leq \frac{\beta^{*}}{\alpha}\left(\sum_{T \in \mathcal{T}_{h}} \gamma_{T} h_{T}^{2 r+1}|u|_{H^{r+1}(S(T))}^{2}\right)^{1 / 2}
$$

where $0 \leq r \leq k$ and $\beta^{*}=\beta^{*}\left(\mu, k, \mu_{1}\right)$. 


\section{References}

1. C. Baiocchi, F. Brezzi, and L.P. Franca, Virtual bubbles and GaLS. CMAME, 105 (1993) 125-142.

2. F. Brezzi, L.P. Franca, T.J.R. Hughes, and A. Russo, $b=\int g$. CMAME, 145 (1997) 329-339.

3. F. Brezzi, L.P. Franca, and A. Russo, Further considerations on residual free bubbles for advective-diffusive equations. (To appear in CMAME).

4. F. Brezzi, T.J.R. Hughes, L.D. Marini, A. Russo, and E. Süli, A priori error analysis of a finite element method with residual-free bubbles for advection-dominated equations. Oxford University Computing Laboratory. Technical Report, 98/07, 1998.

5. F. Brezzi, L.D. Marini, and A. Russo, Applications of pseudo residual-free bubbles to the stabilization of convection-diffusion problems. (To appear in CMAME).

6. F. Brezzi and A. Russo, Choosing bubbles for advection-diffusion problems. Mathematical Models and Methods in Applied Science, 4 (1994) 571-587.

7. A.N. Brooks and T.J.R. Hughes, Streamline upwind/Petrov-Galerkin formulations for convection dominated flows with particular emphasis on the incompressible Navier-Stokes equations. Computer Methods in Applied Mechanics and Engineering, 82 (1982) 199-259.

8. P.G. Ciarlet. The Finite Element Method for Elliptic Problems. North-Holland, Amsterdam 1978.

9. L.P. Franca, S.L. Frey and T.J.R. Hughes, Stabilized finite element methods: I. Applications to advective-diffusive model. CMAME, 95 (1992) 253-276.

10. L.P. Franca and A. Russo, Deriving upwinding, mass lumping and selective reduced integration by residual-free bubbles. Applied Mathematics Letters, 9 (1996) 83-88.

11. L.P. Franca, A. Nesliturk, and M. Stynes, On the stability of residual-free bubbles for convection-diffusion problems and their approximation by a two-level finite element method. Technical report No. 119, Department of Mathematics, University of Colorado at Denver, 1998.

12. T.J.R. Hughes, Multiscale phenomena: Green's functions, the Dirichlet to Neumann formulation, subgrid scale models, bubbles and the origins of stabilised methods. CMAME, 127 (1995) 387-401.

13. T.J.R. Hughes, G. Feijoo, L. Mazzei, and Jean-Baptiste Quincy, The variational multiscale method - A paradigm for computational mechanics, CMAME, (to appear).

14. C. Johnson, U. Nävert, and J. Pitkäranta, Finite element methods for linear hyperbolic problems, CMAME, 45 (1984) 285-312.

15. H.-G. Roos, M. Stynes, and L. Tobiska, Numerical methods for singularly perturbed differential equations: convection diffusion and flow problems, Springer-Verlag, 1996.

16. A. Russo, Bubble stabilization of finite element methods for the linearized incompressible Navier-Stokes equations, CMAME, (1996).

17. L. Tartar, Remarks on some interpolation spaces. In: J.-L. Lions and C. Baiocchi (Eds.), Boundary Value Problems for Partial Differential Equations and Applications. Masson, 1993, 229-252.

18. H. Triebel, Interpolation Theory, Function Spaces and Differential Operators. VEB Deutscher Verlag der Wissenschaften. Berlin, 1978.

19. R. Verfürth, Error estimates for some quasi-interpolation operators. Technical report. Department of Mathematics, Universität Bochum, 1997. (To appear in $M_{2} A N$ ). 